\title{
V1223 Sgr and the long-term activity of the intermediate polars
}

\section{Vojtěch Šimon*}

Astronomical Institute, The Czech Academy of Sciences, 25165 Ondřejov, Czech Republic

Czech Technical University in Prague, Faculty of Electrical Engineering, Prague, Czech

Republic

E-mail: simon@asu.cas.cz

This paper shows that the long-term activity of the intermediate polar (IP) V1223 Sgr contains clusters of the low-state episodes, sometimes even with a well-defined recurrence time. This can be caused by a migration of star spots through the L1 point because of the differential rotation of the donor. Large decreases of intensity in the $15-50 \mathrm{keV} \mathrm{X-ray} \mathrm{band} \mathrm{accompany} \mathrm{the} \mathrm{optical}$ relatively shallow low states of V1223 Sgr. These X-ray variations are thus the results of the decreases of the mass inflow rate to the disk from the donor. Absolute optical magnitudes $M_{O}$ of IPs show that most of these systems lie between those of dwarf novae (DNe) in outburst and quiescence, so their disks are exposed to a thermal-viscous instability (TVI). The value of $M_{O}$ of V1223 Sgr in the high state is one of the brightest among IPs and is close to that of DNe in outburst. This suggests that the disk of V1223 Sgr is in the hot (ionized) state. The values of $M_{O}$ of the IPs V426 Oph and TV Col lie in the region in which a TVI should be present. Indeed, the optical brightness of V426 Oph is highly unstable on superorbital timescales. Short outbursts with similar decay rates start from a highly variable baseline level. TV Col shows a gradual evolution of brightness on timescale of hundreds of days. The outbursts shorter than a day are superimposed on it. GK Per with a very long orbital period shows long dwarf-nova outbursts with the rise of the optical intensity accompanied by a brightening in the hard X-ray band.

The Golden Age of Cataclysmic Variables and Related Objects - III,

7-12 September 2015

Palermo, Italy

* Speaker. 


\section{Introduction}

In cataclysmic variables (CVs) with mildly magnetized white dwarfs (WDs) ( $B \approx 10^{6}$ Gauss), the so-called intermediate polars (IPs) [31], the accretion disk is often present, but its inner region is truncated by the magnetic field of the WD. Accretion curtains connecting the inner disk region and the regions at the magnetic poles (polar caps) of the WD cause an almost radial inflow onto the WD. Thermal emission of the accretion disk dominates in the optical and ultraviolet regions. Bremsstrahlung is emitted by the accretion columns at the polar caps. The defining sign of IPs is the presence of two periods in the CV: the orbital period $P_{\text {orb }}$ and the spin period of the WD $\left(P_{\text {spin }}<P_{\text {orb }}\right)$.

\section{The long-term activity of V1223 Sgr}

V1223 Sgr is an IP [31] which displays strong long-term activity with episodes of low states (e.g. [7]). Fig. 1 shows the light curve of this long-term activity, spanning 78 years (adapted from [24]). It consists of several data sets: archival photographic plates of [7] and the Bamberg Observatory, and CCD ASAS-3 [18].

Since the observations of the long-term activity are often sampled, with various gaps, it is necessary to use the proper method for obtaining the parameters and properties of this activity. The statistical distribution of brightness and its parameters (the standard deviation, skewness) may not be very distorted by the sampling of the data (if a long time segment is mapped). This method can be used for a description of the properties of the long-term activity of CVs and also for resolving among the types of CVs even in the sampled photometric data. This approach is therefore helpful for describing the properties of the long-term activity of CVs.

Examples of these histograms of brightness are shown in Fig. 2. Notice that the low-state episodes tend to occur in clusters. Their profiles are dominated by a broad bump with the peakto-peak amplitude of more than $1 \mathrm{mag}(B)$. The high state is not any uniquely defined level of brightness. Two groups of the low states represent tails from such bumps rather than forming specific levels of brightness. The fuzzy boundaries of the bright side of the statistical distributions of brightness show that the system cannot find equilibrium of the mass transfer even in the high state.

Variograms allow to search for characteristic time scales or quasi-periods which extend only for several epochs. Variogram characterizes the spatial continuity or roughness of a data set ${ }^{1}$. In the case of V1223 Sgr, variograms revealed a dramatic change of the recurrence timescale of the low-state episodes between two clusters of the low-state episodes (see [24] for details). I interpret it as a change of the stellar activity of the donor in V1223 Sgr.

The ASAS-3 data, mapping the activity of V1223 Sgr between the years 1999 and 2009 (Fig. 1), show a recurrence in the episodes of the low states (the second cluster of the low-state episodes). Period search using the phase dispersion minimization (PDM) code [26], included in Peranso $^{2}$, detected a period of 1092 days. This length was discordant with the typical recurrence times present in the first cluster. The profile of the ASAS-3 light curve and the depth of the low

\footnotetext{
${ }^{1}$ www.goldensoftware.com/variogramTutorial.pdf

${ }^{2}$ www.peranso.com
} 


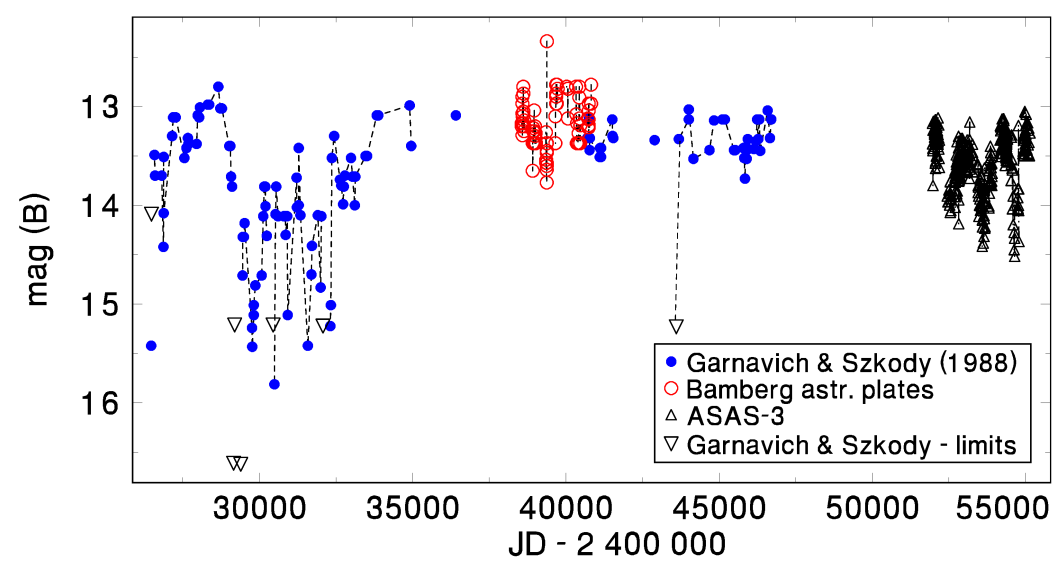

Figure 1: Long-term optical activity of V1223 Sgr. Photographic and CCD observations (ASAS-3 data) were used. All measurements were transformed to the $B$ band and are connected by the lines to guide the eye.

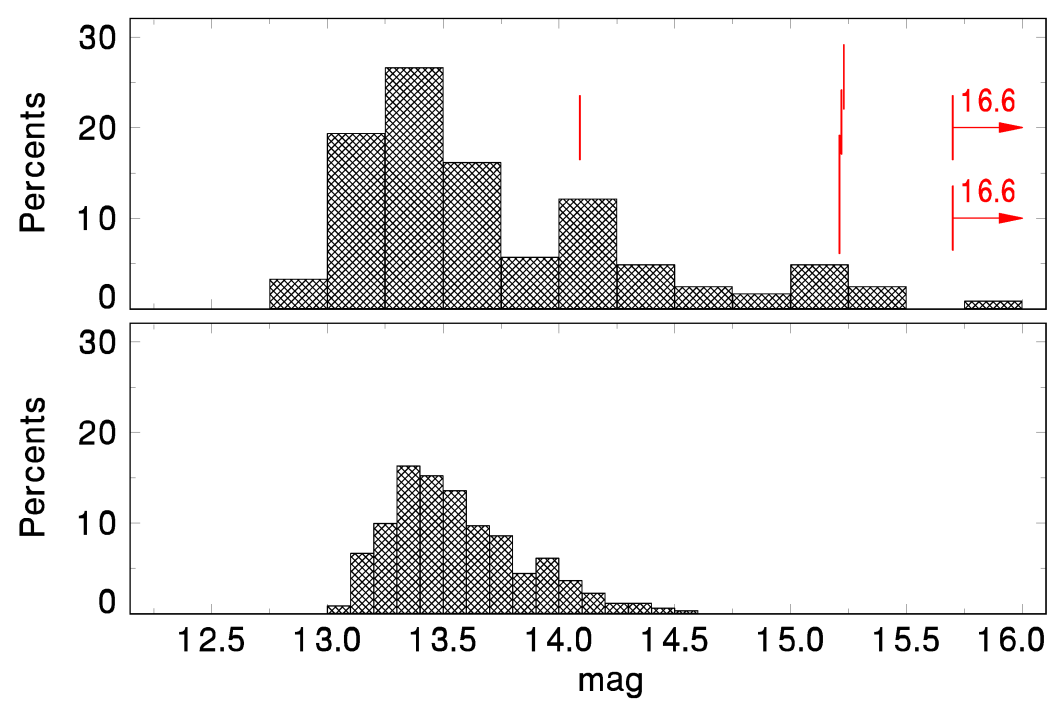

Figure 2: Statistical distribution of brightness of V1223 Sgr. (a) Photographic data of [7]. Bar widths are 0.25 mag. The lines show the upper limits of brightness. (b) Data from ASAS-3. Bar widths are 0.10 mag.

state repeat with good accuracy in the individual epochs. The decrease to the low state occurs on a similar timescale as the rise. The brightness varies relatively gradually during the whole cycle. The episode of the low state is therefore not an isolated event. In my interpretation, this cycle is driven by the changing aspect of some structure (e.g. a sequence of star spots migrating across the nozzle in the L1 point [17]) on the donor. Because of the differential rotation of the tidally locked donor (modeled by [21]), this configuration cyclically influences the mass flow to the accretion disk. More details are in [24].

V1223 Sgr is an X-ray source with a very hard bremsstrahlung X-ray spectrum (e.g. [10]). This spectrum is much harder than that of the novalike MV Lyr in the high state $(<0.5 \mathrm{keV}$ 


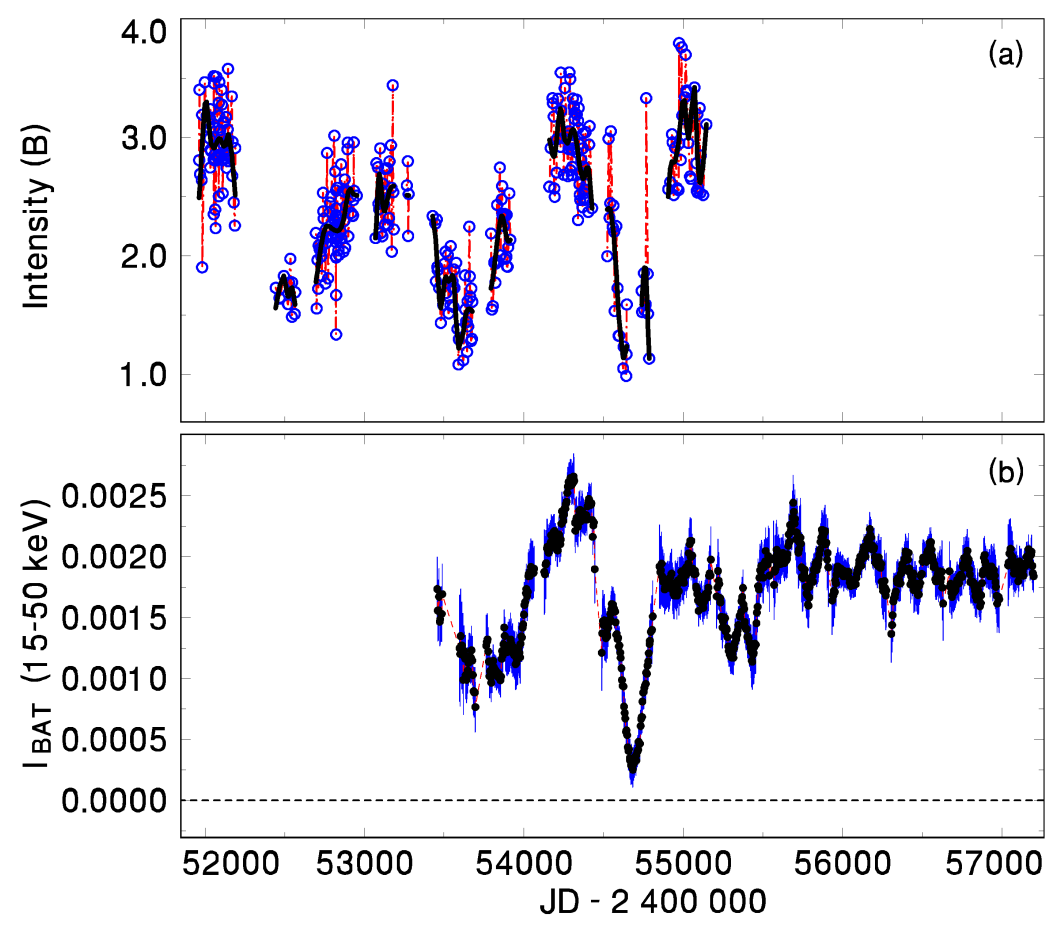

Figure 3: Relation of intensities of V1223 Sgr in the optical and hard X-ray bands. (a) ASAS-3 data [18] transformed to the $B$ band (open circles) (see [24] for details). The solid line represents the fit by the HEC13 code (written by [9], based on the method of [29]). (b) Intensities in the 15-50 keV X-ray band (BAT/Swift). The moving averages are shown. The error bars represent the standard errors.

blackbody emission [8]). V1223 Sgr is therefore observable with BAT/Swift [15] in the 15-50 keV band. Figs. 3 shows that the episodes of the optical low states are caused by decreases of the intrinsic X-ray luminosity. This places constraints on the model of [2], and suggests the decreases of the mass inflow to the disk from the donor with the resulting changes of the rate of accretion onto the WD. Changes of the medium reverberating the $\mathrm{X}$-ray radiation can play a role in modifying the relation of the X-ray and optical luminosities in the subsequent high states. Monitoring with BAT/Swift also shows the evolution of this series of the low-state episodes with time. This series gradually finished and the BAT light curve begun to show only a stable X-ray intensity.

\section{The IPs and their long-term activity}

Absolute optical magnitudes in Fig. 4 show that most IPs lie between those of dwarf novae (DNe) in outburst and quiescence. This enables to assess the conditions in V1223 Sgr in the context of the long-term activity of the IPs. Fig. 4 shows that a thermal-viscous instability of the accretion disk [22] should be present in most IPs. This should lead to DN outbursts modified by a truncation of the inner disk region by the magnetic field of the WD. The model of [1] predicts that these outbursts will be shorter if the inner disk region is truncated by the magnetic field of the WD and if the heating front which switches the disk from the cool to the hot state (outburst) starts in the outer disk region and propagates toward the WD (so if the outburst is of the outside-in type). 


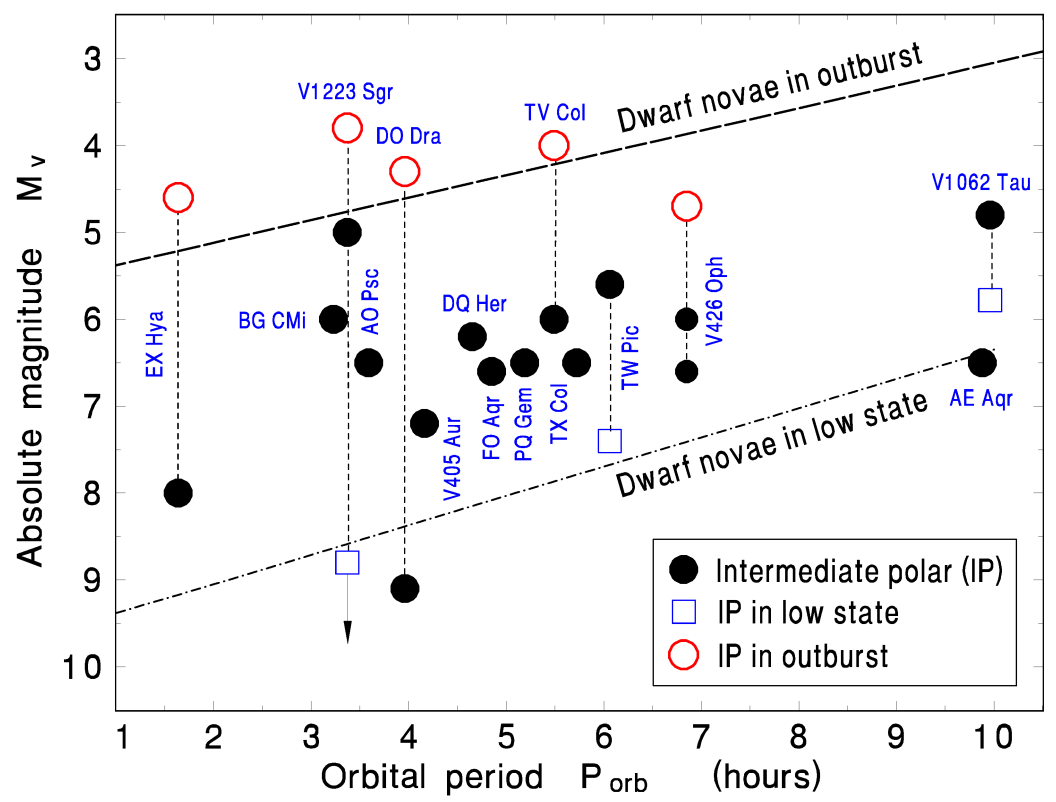

Figure 4: Absolute optical magnitudes of IPs versus their $P_{\text {orb }}$. Outbursts and low states are marked by the empty circles and squares, respectively (most data are from [30] and [31]). GK Per lies out of range. The relation between $P_{\text {orb }}$ and the peak brightness of outbursts of dwarf novae and their quiescent level are according to [30]. The plot is updated from [23].

We can see that the absolute magnitude of V1223 Sgr in the high state is close to that of DNe in outburst. This suggests that the disk is in the hot state. V1223 Sgr sometimes undergoes the low-state episodes when its absolute magnitude can be fainter than that of DNe in quiescence. Nevertheless, the light curve of V1223 Sgr in these low states [7] does not resemble the outbursts in DNe.

GK Per is an IP [32] that has been displaying dwarf nova (DN) outbursts [11]. Its $P_{\text {orb }}=1.99 \mathrm{~d}$ [4] is very long among IPs. The increase of accretion onto the polar caps in a DN outburst leads to an increase of hard X-ray luminosity and to a further hardening of the X-ray spectrum of the bremsstrahlung emission [14]. As I showed in [25], the 1.5-12 keV band (ASM/RXTE [16]) and the 1.5-50 keV band intensities (BAT/Swift [15]) saturate and balance on a plateau during the DN optical outburst [11]. The longer outburst has a longer plateau. The peak X-ray intensities of this series display a significantly narrower range than the optical ones (a factor of about two versus a factor of about eight). This implies a discrepancy between the mass flow through the disk and the production of the X-ray emission via bremsstrahlung at the polar caps of the WD. This discrepancy is the largest in the time of the peak optical intensity when the whole disk (or at least its inner part) is in the hot state and the flow of matter through the disk is the greatest. The saturation of the Xray luminosity in outburst of GK Per cannot be caused by a dominant increase of X-ray absorption. Since the spin modulation of the WD was observed also in a long outburst [6], the accretion onto the caps is not replaced by accretion via boundary layer. In the interpretation in [25], large structural changes of the accreting regions at the magnetic poles of the WD occur during the outburst. A buried shock proposed by [33] for polars is promising also for explaining the X-ray light curves of 

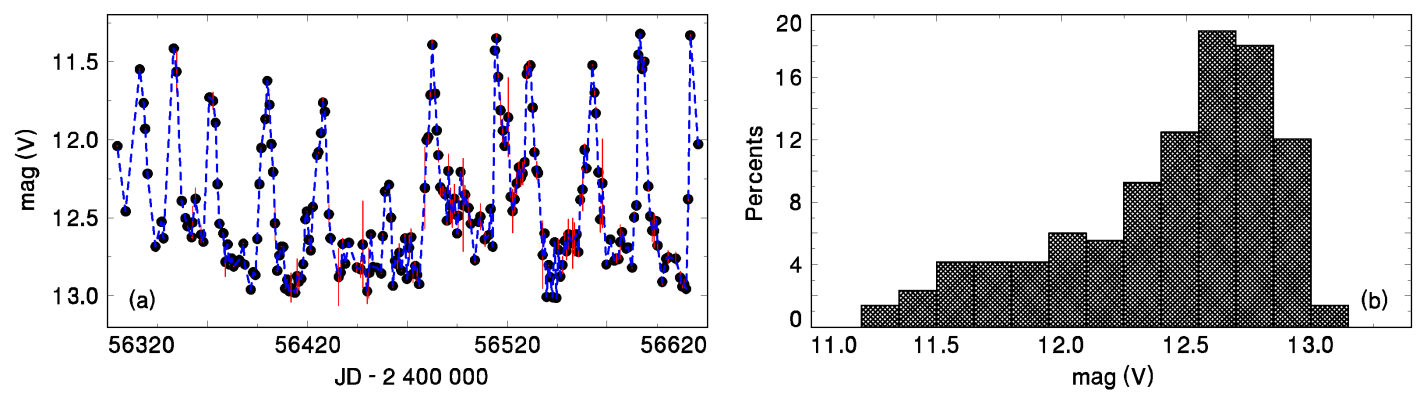

Figure 5: (a) Segment of the optical long-term activity of V426 Oph. One-day means of CCD AAVSO data [12] are used to suppress the intra-night variations. Notice the large-amplitude fluctuations of brightness in quiescence. (b) Statistical distribution of brightness. Notice the very broad peak of the quiescent level.

outbursts of GK Per.

V426 Oph is classified as an IP according to the spin modulation of the WD in X-rays [13]. Its optical brightness (both the mean level and the amplitude) is highly unstable on superorbital timescales. Fig. 5 shows an episode of a highly unstable brightness on superorbital timescales (one-day CCD $V$-band means). Short outbursts with similar decay rates start from a highly variable baseline level. While the DN outbursts are longer for DNe with longer $P_{\text {orb }}$ and a given DN often displays alternating outbursts of two groups according to their length [28], the outbursts of V426 Oph belong only to the short-duration group. This is in accordance with the model of [1] which predicts shorter outbursts if the inner disk region is truncated by the magnetic field of the WD and the outburst is of the outside-in type.

TV Col is a hard X-ray source [3]. It is classified as an IP because it displays several periods [20] [19]. The long-term optical activity shows a gradual evolution on timescale of hundreds of days (Fig. 6a). The profile of these variations was smoothed by the HEC13 code, written by [9] and based on the method of [29]. Fig. 6b shows the statistical distribution of the residuals of this fit. Notice the tail toward the negative values of brightness. This tail can be explained as the short outbursts, whose duration is less that a day. A large segment of such an outburst is shown in Fig. 1 of [20]. The influence of rapid variations of brightness can be lowered if the most deviating residuals are removed. The data sets, in which $20 \%$ or $15 \%$ of such residuals were removed, were fitted again (they can be called the trimmed fits). The results are shown in Fig. 6. The profile of the gradual changes with timescale of hundreds of days is thus smoother.

DO Dra displays only infrequent short outbursts (duration of $\sim 5 \mathrm{~d}$, amplitude of $\sim 5 \mathrm{mag}$, the recurrence time $T_{\mathrm{C}}$ of hundreds of days [23], in accordance with a thermal-viscous instability of the truncated disk modeled by [1]. An increase of intensity of both the thermal emission from the accretion disk (in the optical band) and the bremsstrahlung emission (in the X-ray band) during the outburst is attributed to accretion onto two poles of the magnetized WD [27]. In quiescence, DO Dra displays strong ( $\sim 1 \mathrm{mag}$ ) fluctuations on timescale of weeks and months [12] (much longer than $P_{\text {orb }}$ ), which are independent of the phase between the outbursts. 

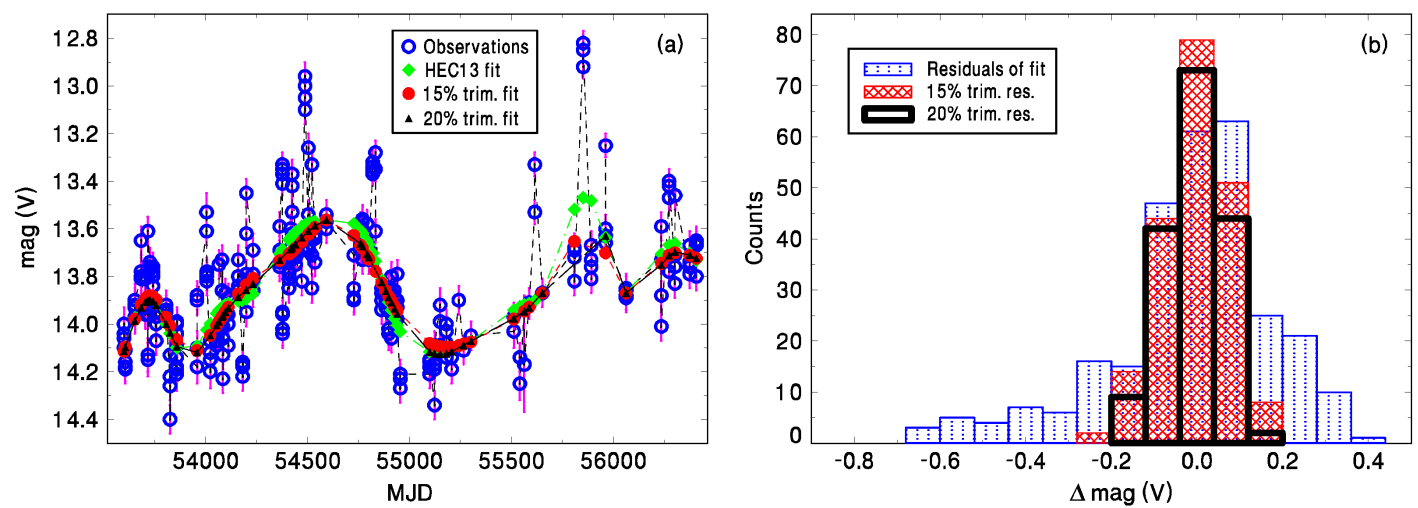

Figure 6: (a) Segment of the optical long-term activity of TV Col (data from Catalina Real-time Transient Survey [5]). (b) Statistical distribution of the residuals of the fits. Notice the tail toward the negative values of brightness. See the text for details.

\section{Conclusions}

Absolute optical magnitudes of IPs (determined mainly by the luminosity of thermal emission of the disk) are often between those of DNe in outburst and quiescence. These IP disks are thus in the zone in which they are exposed to a thermal-viscous instability. Indeed, some of these IPs show a strong activity on long time-scales if they are monitored. Short outbursts (shorter than in "non-magnetic" CVs) of these systems are common. This can be explained if the inner disk region is truncated by the magnetic field of the WD. Brightness of IPs between the outbursts is often significantly variable on super-orbital timescales (but independent on the phase between the outbursts).

Absolute optical magnitude of V1223 Sgr in the high state is one of the brightest among IPs and is close to that of DNe in outburst. This suggests that the disk of V1223 Sgr is in the hot (ionized) state (in variance with many other IPs). The long-term brightness variations of V1223 Sgr can be explained by the activity of the donor, which causes the largely variable mass inflow rate into the disk.

Changes of the hard X-ray intensity (bremsstrahlung emission from the polar caps) of IPs on long timescales are often correlated with those of the optical intensity of the disk. In some IPs, they can be caused by the variations of the mass inflow rate to the disk from the donor, in some other IPs by the thermal-viscous instability of the accretion disk.

Acknowledgments This study was supported by grants 13-39464J and 13-33324S provided by the Grant Agency of the Czech Republic. This research has made use of the observations provided by the ASM/RXTE team and public data from Swift/BAT transient monitor provided by the Swift/BAT team. This research has also made use of the observations from the ASAS project, Catalina Transient Survey, AAVSO International database (USA) and the AFOEV database (France). I thank the variable star observers worldwide. I also thank Prof. P. Harmanec for providing me with the code HEC13. The Fortran source version, compiled version and brief instructions on how to use the program can be obtained at astro.troja.mff.cuni.cz/ftp/hec/HEC13/ 


\section{References}

[1] L. Angelini, \& F. Verbunt, MNRAS, 238, 697 (1989).

[2] K. Beuermann, et al., $A \& A, 4$ 419, 291 (2004).

[3] Cooke, B. A., et al., MNRAS, 182, 489 (1978).

[4] D. Crampton, et al., ApJ, 300, 788 (1986).

[5] A. J. Drake, et al., ApJ, 696, 870 (2009).

[6] P. A. Evans, et al., MNRAS, 399, 1167 (2009).

[7] P. Garnavich, \& P. Szkody, PASP, 100, 1522 (1988).

[8] J. Greiner, $A \& A$, 336, 626 (1998).

[9] P. Harmanec, 1992, http://astro.troja.mff.cuni.cz/ftp/hec/HEC13/

[10] T. Hayashi, \& M. Ishida, MNRAS, 441, 3718 (2014).

[11] Henden, A. A., 2014, Obs. from the AAVSO Int. Database, www.aavso.org

[12] Henden, A. A., 2015, Obs. from the AAVSO Int. Database, www.aavso.org

[13] L. Homer, et al., ApJ, 610, 991 (2004).

[14] M. Ishida, et al., MNRAS, 254, 647 (1992).

[15] H. A. Krimm, et al., ApJS, 209, 14 (2013).

[16] A. M. Levine, et al., ApJ, 469, L33 (1996).

[17] M. Livio, \& J. E. Pringle, ApJ, 427, 956 (1994).

[18] G. Pojmanski, AcA, 47, 467 (1997).

[19] V. R. Rana, et al., AJ, 127, 489 (2004).

[20] A. Retter, et al., MNRAS, 340, 679 (2003).

[21] E. T. Scharlemann, ApJ, 253, 298 (1982).

[22] J. Smak, AcA, 34, 161 (1984)

[23] V. Šimon, $A \& A, \mathbf{3 6 0}, 627$ (2000).

[24] V. Šimon, New Astronomy, 33, 44 (2014).

[25] V. Šimon, $A \& A$, 575, A65 (2015).

[26] R. F. Stellingwerf, ApJ, 224, 953 (1978).

[27] P. Szkody, et al., AJ, 123, 413 (2002).

[28] J. van Paradijs, $A \& A$, 125, L16 (1983).

[29] J. Vondrák, Bull. Astron. Inst. Czechosl., 20, 349 (1969).

[30] B. Warner, MNRAS, 227, 23 (1987).

[31] Warner, B., Cataclysmic Variable Stars, Cambridge Univ. Press, Cambridge (1995).

[32] M. G. Watson, et al., MNRAS, 212, 917 (1985).

[33] D. T. Wickramasinghe, \& L. Ferrario, NewAR, 44, 69 (2000). 